\title{
Exact path-integral evaluation of locally interacting systems: The subtlety of operator ordering
}

\author{
Nobuhiko Taniguchi* \\ Physics Division, Faculty of Pure and Applied Sciences, University of Tsukuba, Tennodai Tsukuba 305-8571, Japan
}

(Received 18 January 2017; published 10 October 2017)

\begin{abstract}
We discuss how one calculates the coherent path integrals for locally interacting systems, where some inconsistencies with exact results have been reported previously. It is shown that the operator ordering subtlety that is hidden in the local interaction term modifies the Hubbard-Stratonovich transformation in the continuous time formulation, and it helps reproduce known results by the operator method. We also demonstrate that many-body effects in the strong interaction limit can be well characterized by the free-particle theory that is subject to annealed random potentials and dynamical gauge (or phase) fields. The present treatment expands the conventional paradigm of the one-particle description, and it provides a simple, viable picture for strongly correlated materials of either bosonic or fermionic systems.
\end{abstract}

DOI: 10.1103/PhysRevA.96.042105

\section{INTRODUCTION}

The path-integral formulation [1,2] has been widely used in many areas of physics and has now become an indispensable tool in formulating, investigating, and understanding quantum physics. Its variant, the coherent-state path integral $[3,4]$, is particularly useful and versatile for analyzing quantum many-body theories where the Hamiltonian is expressed in normal-ordered products of creation and annihilation operators [5]. It helps us handle either bosons or fermions, perform a perturbational expansion, treat nonperturbative contributions like topological effects, and grasp relevant physics intuitively.

The downside of the path-integral approach is that its direct evaluation tends to demand more effort than that of the operator method. Even for noninteracting quadratic Hamiltonians, great care is needed to tackle the operator ordering subtlety or a seemingly divergent determinant. The situation gets exacerbated for interacting systems, even for the simplest possible interacting system, namely the one-site Bose-Hubbard model. When one uses the time-continuous coherent-state path integral to evaluate, say, $\operatorname{Tr}\left[e^{-\beta U c^{\dagger} c^{\dagger} c c / 2}\right]$ with a single bosonic field, one may well be deceived into reaching the wrong answer $\sum_{n=0}^{\infty} e^{-\beta U n^{2} / 2}$, instead of the correct one $\sum_{n=0}^{\infty} e^{-\beta U n(n-1) / 2}[6,7]$. The form of discrepancy strongly suggests that the approach may be plagued by some operator ordering subtlety that the quartic term may have. The same problem prevails in many-particle systems with local interaction, either of bosons or of fermions.

In order to remedy this embarrassing situation, Wilson and Galitski [6] surmised that an additional correction is present in the representation of the normal-ordered interaction in a way to reproduce the exact result. Kordas et al. [7] subsequently proposed a possible, consistent redefinition of the coherent-state path-integral formulation that successfully reproduces the correct results of the one-site Bose-Hubbard model. The scheme is nonstandard, though. Starting with a normal-ordered Hamiltonian, they expressed normal-ordered operators in terms of the coordinate-momentum representation (or the Weyl symbol). The procedure is inconvenient and nontrivial when one tries to treat many-particle bosonic

*taniguchi.n.gf@u.tsukuba.ac.jp systems whose degree of freedom is large, not to mention systems that involve many fermions. Because the many-body Hamiltonian is normal ordered, complying with the standard coherent-state formulation has a clear benefit. It is worth understanding what goes wrong in its conventional treatment and finding a simple, reliable way of reaching correct results.

\section{PURPOSE}

In this paper, we reexamine the coherent-state path integral for locally interacting many-body systems where constituent particles are either bosons or fermions. Like the one-site Bose-Hubbard model, the coherent path integral seemingly fails to reproduce the exact results of the partition function and Green's function, if one uses the conventional definition. We scrutinize the evaluation process and identify the cause in the operator ordering subtlety hidden in the interaction term. We find that circumventing that subtlety makes us modify the Hubbard-Stratonovich (HS) transformation. Accordingly, one can readily reproduce the known exact results in the standard definition of the coherent-state path integral. Our discussion focuses on a simple type of local interaction defined in Eq. (1), but the same argument can straightforwardly apply to a more general form of the interaction among mutually commuting operators, while treating the interaction between mutually noncommuting operators is nontrivial (see Appendix D).

Locally interacting models can be viewed as the strong interaction limit of correlated materials where the interaction is much greater than the band width so that each site is effectively isolated. Charge-blocking, many-body Mott physics dominates, and the one-particle picture gets inappropriate. Propagating degrees of freedom responsible for such dynamical gap is elusive [8]. In the process of evaluating the path integral, we will encounter a certain free-particle theory that is subject to dynamical phase fields and random potentials. This supplement to the free-particle theory is of great interest because it tells how the free-particle theory can accommodate nonperturbative many-body correlation. One can describe strongly correlated materials by using emergent gauge field [9]. Because of charge blocking, the phase degree fluctuates dynamically far beyond Gaussian, and so does the gauge field, which is the time derivative of the phase field. In this respect, one may regard the present calculation as a 
concrete example of how to analyze dynamical fluctuations of the emergent gauge field in the strong interaction limit.

\section{LOCALLY INTERACTING SYSTEMS}

\section{A. Model}

We consider a multilevel (or multisite) system of bosonic or fermionic particles $\left(\psi_{\alpha}, \psi_{\alpha}^{\dagger}\right)$, which interact locally. The Hamiltonian is given by

$$
\hat{H}=\sum_{\alpha} \epsilon_{\alpha} \hat{n}_{\alpha}+\frac{U}{2} \hat{N}(\hat{N}-1),
$$

where the label $\alpha$ refer to levels and/or spins, and $\hat{N}=$ $\sum_{\alpha} \hat{n}_{\alpha}=\sum_{\alpha} \psi_{\alpha}^{\dagger} \psi_{\alpha}$ is the total number operator. In spite of the interaction being present, one can exactly calculate thermodynamics and various Green's functions by help of the operator method (see Appendix A). Yet, with the coherent-state path integral, one must be cautious to reach those results.

\section{B. Subtlety disclosed}

We start by revealing a subtlety hidden in the standard manipulation of the coherent path integral. Taking the grand partition function $\Xi_{U}(\mu)=\operatorname{Tr}\left[e^{-\beta(\hat{H}-\mu \hat{N})}\right]$, we can establish the exact identity between $\Xi_{U}(\mu)$ and the noninteracting counterpart $\Xi_{0}(\mu)$ :

$$
\Xi_{U}(\mu)=\int_{-\infty}^{\infty} d[\tilde{\varphi}] e^{-\beta \frac{\tilde{\varphi}^{2}}{2 U}} \Xi_{0}\left(\mu+\frac{U}{2}-i \tilde{\varphi}\right),
$$

which is derived in Eq. (B2). Here $\tilde{\varphi}$ denotes a timeindependent Gaussian variable with variance $U / \beta$ and the measure $d[\tilde{\varphi}]=\sqrt{\beta / 2 \pi U} d \tilde{\varphi}$ includes the normalization. Relation (2) holds for either bosons or fermions. One can see Eq. (2) come from the operator identity [see Eq. (B3)],

$$
e^{-\beta \frac{U}{2} \hat{N}^{2}}=\int_{-\infty}^{\infty} d[\tilde{\varphi}] e^{-\beta \frac{\tilde{\varphi}^{2}}{2 U}-i \beta \tilde{\varphi} \hat{N}}
$$

The formula can be viewed as an operative version of the HS transformation. An important observation is that when we take the coherent path-integral representation of Eq. (3), it contradicts the standard form of the HS transformation. Indeed, the decomposition concerning $e^{-\beta \hat{H}}$ becomes (see Appendix B for the derivation)

$$
\int \mathcal{D}[\psi, \bar{\psi}] e^{-\mathcal{S} / \hbar}=\int \mathcal{D}[\tilde{\phi}] \mathcal{D}[\psi, \bar{\psi}] e^{-\left(\mathcal{S}_{e}+\mathcal{S}_{\phi}\right) / \hbar},
$$

where the Euclidean actions $\mathcal{S}$ and $\mathcal{S}_{e, \phi}$ are defined by

$$
\begin{aligned}
\mathcal{S} & =\sum_{\alpha, \beta} \int_{0}^{\beta \hbar} d \tau \bar{\psi}_{\alpha}\left[\left(\hbar \partial_{\tau}+\epsilon_{\alpha}\right) \delta_{\alpha \beta}+\frac{U}{2} \bar{\psi}_{\beta} \psi_{\beta}\right] \psi_{\alpha}, \\
\mathcal{S}_{e} & =\int_{0}^{\beta \hbar} d \tau \sum_{\alpha} \bar{\psi}_{\alpha}\left(\hbar \partial_{\tau}+\epsilon_{\alpha}-\frac{U}{2}+i \tilde{\phi}\right) \psi_{\alpha}, \\
\mathcal{S}_{\phi} & =\int_{0}^{\beta \hbar} d \tau \frac{\tilde{\phi}^{2}}{2 U} .
\end{aligned}
$$

The above formula differs from the standard HS formula by the presence of $-U / 2$ in $\mathcal{S}_{e}$. It is caused by circumventing the operator ordering subtlety hidden in the standard derivation of the HS transformation (see Appendix C) and tells us to modify the HS transformation, when we comply with the standard definition of the coherent-state path integral. With the modified representation of the interaction, we can readily evaluate the path-integral expression of $\Xi_{U}(\mu)$ by following each step of Appendix B reversely.

In addition to the operator ordering subtlety, the HS transformation has been known to be plagued by the ambiguity in selecting relevant channels [10]. When truncating relevant fluctuations, it causes a serious problem that might give a different physical result. In the present treatment, however, we do not have such a problem, because we carry out the complete integration of the auxiliary fields without any approximation, thanks to the gauge transformation. Moreover, perturbative treatment often brings divergent contributions due to interaction, and it therefore needs an additional renormalization procedure. This is not the case here, because the knowledge of $\Xi_{0}(\mu)$ is sufficient to calculate $\Xi_{U}(\mu)$ exactly.

\section{Green functions}

We now turn our attention to evaluating various one-particle Green functions of locally interacting systems. Below, we use the closed-time path-integral formalism [5,11-14] to formulate real-time correlation functions. We show how we can exactly evaluate those path-integral representations by using a gauge transformation technique [15-18]. Such approach was undertaken in Ref. [18] to investigate the tunneling density of states at Coulomb-blockade peaks of fermionic locally interacting systems, but its exposition is too succinct to clarify the subtlety of the coherent-state path integrals. We demonstrate how the modified HS transformation [Eq. (9) below], which extends Eqs. (4) to include real-time paths, enables us to evaluate them. Later in Sec. III, we will show that they are identical to what are calculated by the operator method. Moreover, we find that Green's functions for a locally interacting system can be connected and determined by the knowledge of noninteracting systems, like the grand partition function [see Eqs. (15) or (20) below].

We define four types of real-time Green's functions,

$$
\left(\begin{array}{cc}
G_{\alpha}(t, 0) & G_{\alpha}^{<}(t, 0) \\
G_{\alpha}^{>}(t, 0) & \tilde{G}_{\alpha}(t, 0)
\end{array}\right)=\frac{1}{i \hbar}\left(\begin{array}{cc}
\left\langle T \psi_{\alpha}(t) \psi_{\alpha}^{\dagger}\right\rangle & \pm\left\langle\psi_{\alpha}^{\dagger} \psi_{\alpha}(t)\right\rangle \\
\left\langle\psi_{\alpha}(t) \psi_{\alpha}^{\dagger}\right\rangle & \left\langle\tilde{T} \psi_{\alpha}(t) \psi_{\alpha}^{\dagger}\right\rangle
\end{array}\right)
$$

where \pm refers to bosonic or fermionic systems and $\langle\cdots\rangle$ is the thermal average specified by chemical potential $\mu$ and the inverse temperature $\beta$. The operator $T$ is the time-ordering operator, while $\tilde{T}$ is the anti-time-ordering one. We can compactly write them by the contour-ordering operator $T_{c}$ along the Keldysh path $\int_{K}$ as

$$
\begin{aligned}
G_{\alpha}\left(t_{1}, t_{2}\right)= & \frac{1}{i \hbar}\left\langle T_{c} \psi_{\alpha}\left(t_{1}\right) \psi_{\alpha}^{\dagger}\left(t_{2}\right)\right\rangle, \\
& =\frac{1}{i \hbar \Xi_{U}} \int \mathcal{D}[\psi, \bar{\psi}] \psi_{\alpha}(1) \bar{\psi}_{\alpha}(2) e^{\frac{i}{\hbar} S},
\end{aligned}
$$

where the path is composed of three segments (see Fig. 1): the forward-going (denoting -) from the initial time $t_{i}$ to the final time $t_{f}$, the backward-going (denoting + ) from $t_{f}$ to $t_{i}$, and the thermal one from $t_{i}$ to $t_{i}-i \beta \hbar$. 


$$
\phi(t)=i \tilde{\underbrace{}_{t_{i}-i \beta \hbar}} \stackrel{t_{i}}{\phi_{-}(t)=\varphi_{-}-\hbar \dot{\theta}_{-}(t)=\varphi_{+}-\hbar \dot{\theta}_{+}(t)} t_{f}
$$

FIG. 1. The Keldysh contour is composed of three segments: the forward-going $t_{i} \rightarrow t_{f}$, the backward-going $t_{f} \rightarrow t_{i}$, and the thermal $t_{i} \rightarrow t_{i}-i \beta \hbar$. The arrows specify the contour ordering. Time arguments of Green's functions reside between $t_{i}$ and $t_{f}$, and the infinite limit of the time span $\Delta t=t_{f}-t_{i} \rightarrow \infty$ is taken. On each segment, one decomposes the auxiliary field $\phi$ into the static zero mode $\varphi$ and the dynamical nonzero mode $\theta$. One can safely gauge away the nonzero mode on the thermal segment.

Since the interaction $U \hat{N}(\hat{N}-1) / 2$ is normal ordered, the action $S$ that appears in the coherent-state path integral becomes

$$
S=\int_{K} \sum_{\alpha, \beta} \bar{\psi}_{\alpha}\left[\left(i \hbar \partial_{t}-\epsilon_{\alpha}\right) \delta_{\alpha \beta}-\frac{U}{2} \bar{\psi}_{\beta} \psi_{\beta}\right] \psi_{\alpha} .
$$

The next step is crucial: We decompose the interaction term via the modified HS transformation along the Keldysh path. The transformation is

$$
e^{-\frac{i}{\hbar} \int_{K} \frac{U}{2} N^{2}(t)}=\int \mathcal{D}[\phi] e^{\frac{i}{\hbar}\left(S_{\phi}+S_{e}\right)}
$$

where

$$
\begin{gathered}
S_{\phi}=\int_{K} \frac{\phi^{2}(t)}{2 U} \\
S_{e}=\int_{K} \sum_{\alpha} \bar{\psi}_{\alpha}\left[i \hbar \partial_{t}-\epsilon_{\alpha}-\phi(t)+U / 2\right] \psi_{\alpha} .
\end{gathered}
$$

The term $U / 2$ is mandatory in $S_{e}$, as in Eq. (4).

After we have managed the operator ordering subtlety, we may follow the observation in Refs. [15-18] to employ the local gauge transformation to absorb most of the effect of $\phi(t)$. To make this work, however, we have to carefully specify the boundary condition: The periodicity of $\phi(t)$ must be imposed on each of the three segments of the Keldysh path, to ensure new field operators ( $\Psi_{\alpha}$ below) to remain canonical. We then decompose $\phi$ fields on each segment into the static zero modes $\varphi=\left(\varphi_{\mp}, i \tilde{\varphi}\right)$, and the dynamical phase fields $\theta(t)=\theta_{\mp}(t)$ satisfying the periodic boundary condition:

$$
\psi_{\alpha}(t)=e^{i \theta(t)} \Psi_{\alpha}(t) ; \quad \phi(t)=\varphi-\hbar \dot{\theta}(t) .
$$

One can safely gauge away the dynamical field on the thermal segment. Now the action becomes

$$
\begin{aligned}
& S_{e}=\int_{K} \sum_{\alpha} \bar{\Psi}_{\alpha}\left[i \hbar \partial_{t}-\epsilon_{\alpha}-\varphi+U / 2\right] \Psi_{\alpha}, \\
& S_{\phi}=\frac{i \beta \hbar}{2 U} \tilde{\varphi}^{2}+\frac{\Delta t}{2 U}\left(\varphi_{-}^{2}-\varphi_{+}^{2}\right)+\int_{K} \frac{\hbar^{2} \dot{\theta}^{2}}{2 U},
\end{aligned}
$$

with $t_{f}-t_{i}=\Delta t$.

The dynamical phase fields may be regarded as compact $U(1)$ gauge fields that commonly emerge in strongly correlated matter [9]. One may examine nonperturbative correlation effect by studying nontrivial field configurations that carry finite winding numbers. In the present approach, the dynamical fields $\theta_{\mp}$ describe the fluctuating part on top of nontrivial field configurations, while $\varphi=\left(\varphi_{ \pm}, i \tilde{\varphi}\right)$ affects the thermodynamics and its dynamics nonperturbatively.

The result of the $\Psi$ integral can be written by the Green's functions multiplied by the grand partition function of the noninteracting particles. We still need to complete the $\varphi$ and $\theta$ integrals, but in isolated systems here, those integrals are found to be decoupled $[15,16,18]$. Symbolically, one can write the result as

$$
G_{\alpha}(1,2)=\frac{1}{\Xi_{U}}\left\langle\Xi^{\varphi} G_{\alpha}^{\varphi}(1,2)\right\rangle_{\varphi}\left\langle e^{i \theta(1)} e^{-i \theta(2)}\right\rangle_{\theta},
$$

where $\langle\cdots\rangle_{\varphi}$ refers to the Gaussian average over the three static Gaussian variables $\left(\varphi_{\mp}, \tilde{\varphi}\right)$, while $\langle\cdots\rangle_{\theta}$ refers to the path integration over dynamical $\theta$. The explicit forms of $\Xi^{\varphi}$ and $G_{\alpha}^{\varphi}$ are nothing but the noninteracting ones, $\Xi_{0}$ and $G_{0, \alpha}$, where

$$
\begin{gathered}
\Xi_{0}^{\varphi}=\Xi_{0}\left(\left\{\epsilon_{\alpha}^{\varphi}, \mu^{\varphi}\right\}\right)=\prod_{\alpha}\left[1 \mp e^{-\beta\left(\epsilon_{\alpha}^{\varphi}-\mu^{\varphi}\right)}\right]^{\mp 1}, \\
G_{\alpha}^{\varphi}(t, 0)=G_{0, \alpha}\left(t ;\left\{\epsilon_{\alpha}^{\varphi}, \mu^{\varphi}\right\}\right),
\end{gathered}
$$

with incorporating $\varphi$ dependence by shifting $\epsilon_{\alpha}$ and $\mu$ by

$$
\epsilon_{\alpha}^{\varphi}=\epsilon_{\alpha}-\frac{U}{2}+\varphi_{c} ; \quad \mu^{\varphi}=\mu-i \tilde{\varphi}+\varphi_{c}-\frac{i \Delta t}{\beta \hbar} \varphi_{q} .
$$

Here the convention $\varphi_{c}=\left(\varphi_{-}+\varphi_{+}\right) / 2$ and $\varphi_{q}=\varphi_{-}-\varphi_{+}$ is used. When we recover the Keldysh structure, the part $\left\langle e^{i \theta(1)} e^{-i \theta(2)}\right\rangle_{\theta}$ in Eq. (15) means the contour-ordered vertex correlator. We can calculate it as the action regarding $\theta$ is free particle with mass $U / \hbar^{2}$. Though local fluctuation $\left\langle\theta^{2}(t)\right\rangle$ diverges, it is finite and equal to

$$
\left\langle T_{c} e^{-i \theta(t)} e^{i \theta(0)}\right\rangle_{\theta}=\left(\begin{array}{ll}
e^{-\frac{i U}{2 \hbar}|t|} & e^{\frac{i U}{2 \hbar} t} \\
e^{-\frac{i U}{2 \hbar} t} & e^{\frac{i U}{2 \hbar}|t|}
\end{array}\right) .
$$

Combining all the above, we can evaluate exactly all oneparticle Green's functions for locally interacting systems.

Let us briefly illustrate how it operates in practice. The lesser component of Eq. (15) gives

$$
G_{\alpha}^{<}(t, 0)=\frac{1}{\Xi_{U}}\left\langle\Xi_{0}^{\varphi} G_{\alpha}^{\varphi,<}(t, 0)\right\rangle_{\varphi} e^{\frac{i U}{2 \hbar} t},
$$

and the noninteracting lesser Green's function is

$$
G_{\alpha}^{\varphi,<}(t, 0)= \pm \frac{e^{-\frac{i}{\hbar} \epsilon_{\alpha}^{\varphi} t}}{i \hbar} n_{\alpha}^{\varphi}
$$

The occupation $n_{\alpha}^{\varphi}=\left\langle\hat{n}_{\alpha}\right\rangle$ has to be determined by the partition function $\Xi^{\varphi}$ via the standard relation

$$
n_{\alpha}^{\varphi}=-\frac{1}{\beta} \frac{\partial}{\partial \epsilon_{\alpha}} \ln \Xi_{0}^{\varphi} .
$$

It means that $G_{\alpha}^{<}(t, 0)$ of locally interacting systems is expressed in a form of the annealed average over three random (static) Gaussian variables $\varphi=\left(\varphi_{\mp}, \tilde{\varphi}\right)$ :

$$
G_{\alpha}^{<}(t, 0)=\mp \frac{1}{i \hbar \Xi_{U}}\left\langle e^{-\frac{i}{\hbar}\left(\epsilon_{\alpha}^{\varphi}-\frac{U}{2}\right) t} \frac{\partial \Xi_{0}^{\varphi}}{\beta \partial \epsilon_{\alpha}}\right\rangle_{\varphi} .
$$


We can likewise find the greater Green's function,

$$
G_{\alpha}^{>}(t, 0)=\frac{1}{i \hbar \Xi_{U}}\left\langle e^{-\frac{i}{\hbar}\left(\epsilon_{\alpha}^{\varphi}+\frac{U}{2}\right) t}\left[\Xi_{0}^{\varphi} \mp \frac{\partial \Xi_{0}^{\varphi}}{\beta \partial \epsilon_{\alpha}}\right]\right\rangle_{\varphi} .
$$

From these results of $G_{\alpha}^{<}$and $G_{\alpha}^{>}$, we can construct all the other one-particle Green's functions.

\section{EQUIVALENCE TO THE OPERATOR METHOD}

We now check that the results Eqs. (23) and (24) actually reproduce the Green's functions evaluated by the operator method in Appendix A. To see it, we expand $\Xi_{0}^{\varphi}$ in terms of the canonical partition function $Z_{N}$ of nonshifting levels $\epsilon_{\alpha}$,

$$
\Xi_{0}^{\varphi}=\sum_{N=0}^{\infty} Z_{N} e^{N \beta\left(\mu+\frac{U}{2}-i \tilde{\varphi}-\frac{i \Delta t}{\beta \hbar} \varphi_{q}\right)}
$$

We find that the integration over $\varphi_{q}$ simply enforces $\varphi_{c} / U$ to non-negative integers $N$ in the limit of $\Delta t \rightarrow \infty$ :

$$
\int d\left[\varphi_{q}\right] e^{\frac{i \Delta t}{\hbar U} \varphi_{c} \varphi_{q}} e^{-\frac{i \Delta t}{\hbar} \varphi_{q} N}=\delta\left(\varphi_{c}-U N\right) .
$$

Accordingly, we may say that $\varphi_{c} / U$ plays a role of winding numbers of the emergent compact gauge field configuration; a naive saddle-point (or Hartree-Fock) approximation regarding $\varphi$ misses such nonperturbative contribution. We need to take account of all the contribution of $N$ on principle (see Ref. [18] for its implication on the tunneling density of states). By completing the remaining Gaussian average over $\tilde{\varphi}$, we organize the result as

$$
\begin{aligned}
G_{\alpha}^{<}(t, 0) & =\frac{ \pm 1}{i \hbar} \sum_{N=0}^{\infty} e^{-\frac{i}{\hbar}\left[\epsilon_{\alpha}+U(N-1)\right] t} n_{\alpha \mid N}, \\
G_{\alpha}^{<}(\varepsilon) & =\mp 2 i \pi \sum_{N=0}^{\infty} n_{\alpha \mid N} \delta\left[\varepsilon-\epsilon_{\alpha}-U(N-1)\right] .
\end{aligned}
$$

Here we have introduced the quantity $n_{\alpha \mid N}$, the "fractional parentage" of the occupation number onto the fixed $N$. It is defined by

$$
n_{\alpha \mid N}=-\frac{1}{\beta \Xi_{U}} \frac{\partial Z_{N}}{\partial \epsilon_{\alpha}} e^{\beta N \mu-\beta \frac{U}{2} N(N-1)},
$$

and satisfies $\left\langle\hat{n}_{\alpha}\right\rangle=\sum_{N=0}^{\infty} n_{\alpha \mid N}$. Similarly, we find the greater Green's function to be

$$
\begin{aligned}
G_{\alpha}^{>}(t, 0) & =\frac{1}{i \hbar} \sum_{N=0}^{\infty} e^{-\frac{i}{\hbar}\left(\epsilon_{\alpha}+U N\right) t} p_{\alpha \mid N}, \\
G_{\alpha}^{>}(\varepsilon) & =-2 i \pi \sum_{N=0}^{\infty} p_{\alpha \mid N} \delta\left(\varepsilon-\epsilon_{\alpha}-U N\right),
\end{aligned}
$$

by using $p_{\alpha \mid N}$, the fractional parentage of the hole occupation onto a fixed $N$, defined by

$$
p_{\alpha \mid N}=\frac{1}{\Xi_{U}}\left[Z_{N} \mp \frac{1}{\beta} \frac{\partial Z_{N}}{\partial \epsilon_{\alpha}}\right] e^{\beta N \mu-\beta \frac{U}{2} N(N-1)} .
$$

The spectral function $\rho_{\alpha}(\varepsilon)$ is straightforwardly calculated as

$$
\begin{aligned}
\rho_{\alpha}(\varepsilon)= & \sum_{N=0}^{\infty}\left\{p_{\alpha \mid N} \delta\left(\varepsilon-\epsilon_{\alpha}-U N\right)\right. \\
& \left.\mp n_{\alpha \mid N} \delta\left[\varepsilon-\epsilon_{\alpha}-U(N-1)\right]\right\} .
\end{aligned}
$$

In these forms, one can confirm the equivalence with the ones by the operator method in Appendix A.

\section{DISCUSSION}

We have shown that we can treat a locally interacting system correctly using the standard definition of the coherent path integral. The results are connected with their noninteracting counterpart. [See Eqs. (2) for the partition function, and Eqs. (23) and (24) for Green's functions.] The relation (2) shows that the thermodynamics of a locally interacting system is exactly equivalent to the annealed average of the noninteracting Hamiltonian with random imaginary potential $\tilde{\varphi}$. Such simple correspondence, however, cannot be held for Green's functions (23) and (24) - they are still written by a free-particle model under the influence of static random fields, as is seen in Eq. (15), but we can assign no single random Hamiltonian for its dynamics, because three independent random variables are needed: $\varphi_{\mp}$ along the two real-time paths and $\tilde{\varphi}$ on the thermal path. We stress that this supplement to the free-particle theory can fully capture various many-body characteristics like atomic correlations, nonrigid bands, and asymmetry of particle and hole excitations. While a spectral function in the conventional one-particle and/or quasiparticle picture has only a single peak, the function $\rho_{\alpha}(\varepsilon)$ of Eq. (31) has multiple peaks with different weights at $\varepsilon=\epsilon_{\alpha}+U N$. At those energies, the retarded self-energy diverges and the retarded Green's function vanishes, which signals the demise of the quasiparticle picture [8].

To treat the nonperturbative many-body effect, it is important to take account of two aspects: discreteness of the particle number and large phase fluctuations beyond quadratic order. They are closely related. We can implement discreteness of $N$ by compacting the conjugate phase $\Theta$ modulo $2 \pi$ (satisfying $[\hat{N}, \hat{\Theta}]=i$ ). The nonpositive nature of $N$ makes $\Theta$ non-Hermite [19]. Since the phase $\Theta(t)$ couples linearly with $\dot{N}(t)$, we may take the HS field $\phi(t)$ as $\phi(t)=\hbar \dot{\Theta}(t)$. It means that we need to treat fluctuations of $\phi(t)$ consistently by respecting such nontrivial nature of $\Theta$. A common practice after introducing the HS field $\phi(t)$ is to complete the quadratic integration over the field $\left(\psi_{\alpha}, \bar{\psi}_{\alpha}\right)$ and then to take the saddle-point approximation regarding $\phi$. Assuming a uniform solution $\phi(t)=\varphi_{s p}$, one can determine the self-consistent saddle-point solution $\varphi_{s p}$ by the average number $\langle\hat{N}\rangle=\varphi_{s p} / U$ in that approximation. This contrasts with the exact locking of $\varphi_{c} / U$ to non-negative integers in Eq. (26). A physical picture given by the saddle-point approximation is fundamentally wrong, having no dynamical gap generation and retaining the noninteracting Fermi-Dirac form of the occupation $\left\langle n_{\alpha}\right\rangle$. We find the gauge transformation technique is effective to incorporating many-body effects. Without any additional ansatz of the slave particle, one can describe many-body charge-blocking physics. 
In hindsight, it is because the local occupation number is conserved that one can solve locally interacting systems exactly. When we couple a locally interacting system linearly with external environments (reservoirs), the local occupation is no longer conserved, and the integrals over $\varphi$ and $\theta$ are coupled unlike Eq. (15). It seems unlikely that we can complete the remaining path integrals exactly. Nevertheless, the present analysis of path integrals provides a useful and systematic means to describe the local strong correlation that perturbation theory cannot treat. In a quantum dot coupled to the leads, two types of strongly correlated phenomena are known to emerge: the Coulomb blockade (or charge-blocking due to correlation) and the Kondo physics [20]. When we surmise a decoupling approximation in evaluating the $\varphi$ and $\theta$ integrals as in Eq. (15), repeating the same calculation leads us to the spectral function that is similar to Eq. (31). The only difference is that the $\delta$ functions in Eq. (31) now acquire finite width due to the coupling with the reservoirs. It corresponds to the spectral function of the Coulomb blockade regime $[21,22]$. It was further suggested that if one implements a self-consistent decoupling scheme, one may well understand the Kondo physics [17]. It is interesting to see how such decoupling approximation can be improved by taking account of the compact and non-Hermitian nature of phase fluctuations. Our work in this direction is under way.

\section{SUMMARY}

To summarize, we have demonstrated how one can evaluate the coherent-state path integrals for locally interacting systems, following its standard definition and being aware of the operator ordering subtlety. The results agree with the ones by the operator method. In the process of calculating, we find that locally interacting systems is equivalent to certain free-particle models embellished with dynamical phase as well as static random variables. Since we can view locally interacting models as the strong interaction limit of a wide range of strongly correlated materials, we use such free theories as an alternative yet viable simple description for strongly correlated materials.

\section{ACKNOWLEDGMENT}

The author gratefully acknowledges financial support from JSPS KAKENHI No. 26400382 from MEXT, Japan.

\section{APPENDIX A: CALCULATION VIA THE OPERATOR METHOD}

\section{Grand partition function}

Since the effect of the interaction is to increase the energy by $U N(N-1) / 2$ for fixed- $N$ states, we can express the grand partition function of the Hamiltonian (1) as

$$
\Xi_{U}(\mu)=\operatorname{Tr}\left[e^{-\beta(\hat{H}-\mu \hat{N})}\right]=\sum_{N=0}^{\infty} Z_{N} e^{\beta \mu N-\beta \frac{U}{2} N(N-1)},
$$

where $Z_{N}$ is the canonical partition function of the noninteracting system, defined by

$$
\Xi_{0}(\mu)=\sum_{N=0}^{\infty} Z_{N} e^{\beta \mu N}=\prod_{\alpha}\left[1 \mp e^{-\beta\left(\epsilon_{\alpha}-\mu\right)}\right]^{\mp 1} .
$$

The sign $\mp 1$ refers to bosonic or fermionic systems. One can write the explicit form of $Z_{N}$ via the inverse transformation of the above as

$$
Z_{N}=\int_{0}^{2 \pi} \frac{d \theta}{2 \pi} e^{-i N \theta} \Xi_{0}(\mu=i \theta) .
$$

\section{Green's functions}

We can solve exactly various one-particle Green's functions for the locally interacting Hamiltonian (1). The system is not needed to be in thermal equilibrium; a generic stationary state will suffice. A quick way to proceed is to examine the equation of motion for a field operator $\psi_{\alpha}$,

$$
i \hbar \frac{\partial \psi_{\alpha}(t)}{\partial t}=\left(\epsilon_{\alpha}+U \hat{N}\right) \psi_{\alpha}(t),
$$

which is true for either bosonic or fermionic systems. We can immediately solve its time evolution as

$$
\psi_{\alpha}(t)=e^{-\frac{i}{\hbar}\left(\epsilon_{\alpha}+U \hat{N}\right) t} \psi_{\alpha}=\psi_{\alpha} e^{-\frac{i}{\hbar}\left[\epsilon_{\alpha}+U(\hat{N}-1)\right] t} .
$$

With this property, we can calculate various Green's functions. For instance, the lesser and greater Green's functions are found to be

$$
\begin{aligned}
& G_{\alpha}^{<}(t, 0)= \pm \frac{1}{i \hbar}\left\langle\hat{n}_{\alpha} e^{-\frac{i}{\hbar}\left[\epsilon_{\alpha}+U(\hat{N}-1)\right] t}\right\rangle, \\
& G_{\alpha}^{>}(t, 0)=\frac{1}{i \hbar}\left\langle e^{-\frac{i}{\hbar}\left(\epsilon_{\alpha}+U \hat{N}\right) t}\left(1 \pm \hat{n}_{\alpha}\right)\right\rangle,
\end{aligned}
$$

where the average $\langle\cdots\rangle$ refers to some stationary state average. In the energy space, they become

$$
\begin{aligned}
& G_{\alpha}^{<}(\varepsilon)=\mp 2 i \pi\left\langle\hat{n}_{\alpha} \delta\left(\varepsilon-\epsilon_{\alpha}-U(\hat{N}-1)\right\rangle,\right. \\
& G_{\alpha}^{>}(\varepsilon)=-2 i \pi\left\langle\left(1 \pm \hat{n}_{\alpha}\right) \delta\left(\varepsilon-\epsilon_{\alpha}-U \hat{N}\right)\right\rangle .
\end{aligned}
$$

We can construct all other Green's functions using the results of $G_{\alpha}^{<,>}$. The spectral function $\rho_{\alpha}(\varepsilon)=-\operatorname{Im} G_{\alpha}^{R}(\varepsilon) / \pi$ is found to be

$$
\begin{aligned}
\rho_{\alpha}(\varepsilon)= & \left\langle\left(1 \pm \hat{n}_{\alpha}\right) \delta\left(\varepsilon-\epsilon_{\alpha}-U \hat{N}\right)\right. \\
& \left.\mp \hat{n}_{\alpha} \delta\left(\varepsilon-\epsilon_{\alpha}-U(\hat{N}-1)\right)\right\rangle .
\end{aligned}
$$

For fermionic systems, the results take particularly simple forms resembling the free particle, by the property $\hat{n}_{\alpha}^{2}=\hat{n}_{\alpha}$. Indeed, the spectral function becomes

$$
\rho_{\alpha}(\varepsilon)=\left\langle\delta\left(\varepsilon-\epsilon_{\alpha}-U \hat{N}_{\alpha}^{\prime}\right)\right\rangle,
$$

with introducing $\hat{N}_{\alpha}^{\prime}=\hat{N}-\hat{n}_{\alpha}$. All Green's functions likewise have free-fermion forms only with replacing $\epsilon_{\alpha} \mapsto \epsilon_{\alpha}+$ $U \hat{N}_{\alpha}^{\prime}$. When we further assume that the system is in thermal equilibrium with $\mu$ and $\beta$, the Kubo-Martin-Siggia relation makes the average occupation number be characterized by the Fermi-Dirac distribution as

$$
\left\langle\hat{n}_{\alpha}\right\rangle=\left\langle\frac{1}{e^{\beta\left(\epsilon_{\alpha}+U \hat{N}_{\alpha}^{\prime}-\mu\right)}+1}\right\rangle,
$$


though local interaction makes it deviate considerably from the Fermi-Dirac function regarding $\epsilon_{\alpha}-\mu$.

\section{APPENDIX B: DERIVATIONS OF EQS. (2)-(4)}

In this appendix, we present the step-by-step derivations of Eqs. (2)-(4) in the main text. We start with the Gaussian integral formula

$$
e^{-\beta \frac{U}{2} N^{2}}=\int d[\tilde{\varphi}] e^{-\beta \frac{\tilde{\varphi}^{2}}{2 U}-i \beta \tilde{\varphi} N},
$$

where $d[\tilde{\varphi}]$ include the normalization factor and $N$ is just a number. By using the above and Eqs. (A1) and (A2), we immediately prove Eq. (2) as

$$
\begin{aligned}
& \int_{-\infty}^{\infty} d[\tilde{\varphi}] e^{-\beta \frac{\tilde{\varphi}^{2}}{2 U}} \Xi_{0}\left(\mu+\frac{U}{2}-i \tilde{\varphi}\right) \\
& =\int_{-\infty}^{\infty} d[\tilde{\varphi}] e^{-\beta \frac{\tilde{\varphi}^{2}}{2 U}} \sum_{N=0}^{\infty} Z_{N} e^{\beta(\mu+U / 2-i \tilde{\varphi}) N}, \\
& =\sum_{N=0}^{\infty} Z_{N} e^{\beta(\mu+U / 2) N-\beta U N^{2} / 2}=\Xi_{U}(\mu) .
\end{aligned}
$$

We can extend the Gaussian formula (B1) to the operator identity by inserting the complete basis of the occupation number representation $\left|\left\{n_{\alpha}\right\}\right\rangle$ with the total number $N=$ $\sum_{\alpha} n_{\alpha}$ :

$$
\begin{aligned}
e^{-\beta \frac{U}{2} \hat{N}^{2}} & =\sum_{\left\{n_{\alpha}\right\}}\left|\left\{n_{\alpha}\right\}\right\rangle e^{-\beta \frac{U}{2} N^{2}}\left\langle\left\{n_{\alpha}\right\}\right|, \\
& =\sum_{\left\{n_{\alpha}\right\}}\left|\left\{n_{\alpha}\right\}\right\rangle \int d[\tilde{\varphi}] e^{-\beta \frac{\tilde{\varphi}^{2}}{2 U}-i \beta \tilde{\varphi} N}\left\langle\left\{n_{\alpha}\right\}\right|, \\
& =\int d[\tilde{\varphi}] e^{-\beta \frac{\tilde{\varphi}^{2}}{2 U}-i \beta \tilde{\varphi} \hat{N}} .
\end{aligned}
$$

This proves the operator identity (3) in the text. With this identity, we can rewrite the operator $e^{-\beta \hat{H}}$ as

$$
e^{-\beta \hat{H}}=\int d[\tilde{\varphi}] e^{-\beta \frac{\tilde{\varphi}^{2}}{2 U}-\beta \sum_{\alpha}\left(\epsilon_{\alpha}+i \tilde{\varphi}-U / 2\right) \hat{n}_{\alpha}} .
$$

We now represent both sides of Eq. (B4) to establish the modification of the Hubbard-Stratonovich transformation in the coherent-state path integral. Since the Hamiltonian $\hat{H}$ is normal ordered, the left-hand side (LHS) of Eq. (B4) is simply represented as

$$
\begin{gathered}
(\mathrm{LHS})=\int \mathcal{D}[\psi, \bar{\psi}] e^{-\mathcal{S} / \hbar}, \\
\mathcal{S}=\sum_{\alpha, \beta} \int_{0}^{\beta \hbar} d \tau \bar{\psi}_{\alpha}\left[\left(\hbar \partial_{\tau}+\epsilon_{\alpha}\right) \delta_{\alpha \beta}+\frac{U}{2} \bar{\psi}_{\beta} \psi_{\beta}\right] \psi_{\alpha} .
\end{gathered}
$$

Now we can express the right-hand side (RHS) of Eq. (B4) as

$$
\begin{aligned}
(\mathrm{RHS}) & =\int d[\tilde{\varphi}] \int \mathcal{D}[\psi, \bar{\psi}] e^{-\frac{\beta}{2 U} \tilde{\varphi}^{2}-\mathcal{S}_{e} / \hbar} \\
& =\int \mathcal{D}[\theta] \int d[\tilde{\varphi}] \int \mathcal{D}[\psi, \bar{\psi}] e^{-\frac{\beta}{2 U} \tilde{\varphi}^{2}-\mathcal{S}_{1} / \hbar-\mathcal{S}_{\theta} / \hbar}
\end{aligned}
$$

Here the Euclidean action Lagrangian $\mathcal{S}_{1}$ and $\mathcal{S}_{\theta}$ are defined as

$$
\begin{gathered}
\mathcal{S}_{1}=\int_{0}^{\beta \hbar} d \tau \sum_{\alpha} \bar{\psi}_{\alpha}\left(\hbar \partial_{\tau}+\epsilon_{\alpha}-\frac{U}{2}+i \tilde{\varphi}\right) \psi_{\alpha}, \\
\mathcal{S}_{\theta}=\int_{0}^{\beta \hbar} d \tau \frac{\hbar^{2}}{2 U}\left(\partial_{\tau} \theta\right)^{2}
\end{gathered}
$$

and, on Eq. (B8), we have inserted the path integral over bosonic field $\theta$ that satisfies the periodic boundary condition

$$
\int \mathcal{D}[\theta] e^{-\mathcal{S}_{\theta} / \hbar}=1 .
$$

Next, we introduce a new (dynamical) field $\tilde{\phi}(\tau)=\tilde{\varphi}-$ $\hbar \partial_{\tau} \theta(\tau)$ to combine $\tilde{\varphi}$ and $\theta$, and redefine field $\psi_{\alpha}$ to absorb the phase factor. This is the reverse manipulation of the gauge transformation in Refs. [15-18], with the corresponding Jacobian $\mathcal{D}[\theta] d[\tilde{\varphi}]=\mathcal{D}[\tilde{\phi}]$. It enables us to express the right-hand side of Eq. (B4) as

$$
(\mathrm{RHS})=\int \mathcal{D}[\tilde{\phi}] \mathcal{D}[\psi, \bar{\psi}] e^{-\mathcal{S}_{e} / \hbar-\mathcal{S}_{\phi} / \hbar},
$$

where $\mathcal{S}_{e}$ and $\mathcal{S}_{\phi}$ are defined in Eqs. (4c) and (4d); this proves Eqs. (4a)-(4d) in the text.

\section{APPENDIX C: SUBTLETY OF THE HUBBARD-STRATONOVICH DECOUPLING IN THE CONTINUOUS TIME FORMULATION}

We explicitly point out where matters the subtlety of the Hubbard-Stratonovich transformation in the continuous time formulation. Below we write for the one-site bosonic system, but the same argument applies equally to multilevel extension as well as fermionic systems.

We examine how one can evaluate the matrix element $\left\langle z\left|e^{-\frac{i t}{\hbar} \frac{U}{2} \hat{n}^{2}}\right| w\right\rangle$ regarding the bosonic coherent state $|z\rangle=$ $e^{\bar{z} b-b^{\dagger} z}|0\rangle$, with or without the Hubbard-Stratonovich transformation. Direct evaluation of the matrix element leads to

$$
\left\langle z\left|e^{-\frac{i t}{\hbar} \frac{U}{2} \hat{n}^{2}}\right| w\right\rangle=e^{-\frac{1}{2}(\bar{z} z+\bar{w} w)} \sum_{n=0}^{\infty} \frac{(\bar{z} w)^{n}}{n !} e^{-\frac{i t}{\hbar} \frac{U}{2} n^{2}} .
$$

We now decompose the interaction term using the operator identity.

$$
e^{-\frac{i t}{\hbar} \frac{U}{2} \hat{n}^{2}}=\int_{-\infty}^{\infty} d[\varphi] e^{\frac{i t}{\hbar}\left(\frac{\varphi^{2}}{2 U}-\varphi \hat{n}\right)}=\left\langle e^{-\frac{i t}{\hbar} \varphi \hat{n}}\right\rangle_{\varphi},
$$

where $d[\varphi]$ includes the normalization factor and $\langle\cdots\rangle_{\varphi}$ indicates the Gaussian average over $\varphi$. One can check the correctness of this decomposition by putting it on the left-hand side of Eq. (C1) and using the Wick theorem with $\left\langle\varphi^{2}\right\rangle_{\varphi}=$ $\hbar U /(-i t)$ :

$$
\begin{aligned}
& \left\langle z\left|\left\langle e^{-\frac{i t}{\hbar} \varphi \hat{n}}\right\rangle_{\varphi}\right| w\right\rangle \\
& =e^{-\frac{1}{2}(\bar{z} z+\bar{w} w)} \sum_{n=0}^{\infty} \frac{(\bar{z} w)^{n}}{n !} \exp \left[\left\langle\frac{1}{2}\left(\frac{-i t}{\hbar} \varphi n\right)^{2}\right\rangle_{\varphi}\right] .
\end{aligned}
$$

So far so good. Now the subtlety appears when we try to formulate it using the path integral. When we expand the 
expression for infinitesimal time $\delta t$ up to the linear order, we see it behave as

$$
\left\langle z\left|e^{-\frac{i \delta t}{\hbar} \frac{U}{2} \hat{n}^{2}}\right| w\right\rangle \approx e^{-\frac{1}{2}(\bar{z} z+\bar{w} w)} \sum_{n=0}^{\infty} \frac{(\bar{z} w)^{n}}{n !}\left[1-\frac{i \delta t}{\hbar} \frac{U}{2} n^{2}\right] .
$$

Yet, this correct behavior cannot be reproduced when we truncate Eq. (C3) up to the linear order of $\delta t$. The corresponding contribution comes from the quadratic order term proportional to $(\delta t)^{2}\left\langle\varphi^{2}\right\rangle$. In other words, if we naively formulated the continuous-time path integral just by expanding it regarding the linear $\delta t$ and exponentiating it, we would get a wrong result. The missing $U / 2$ term exactly results from this slack manipulation; the use of the modified HubbardStratonovich transformation resolves the issue by avoiding such manipulation carefully.

\section{APPENDIX D: EXTENSIONS OF THE OPERATIVE HUBBARD-STRATONOVICH DECOUPLING}

Our discussion relies on the operator version of the Hubbard-Stratonovich transformation and its path-integral representation. We can generalize the argument to more general forms of interaction composed by a set of mutually commuting operators, such as $\left\{\hat{n}_{\alpha}\right\}$. It is because we can find the simultaneously diagonalized basis $\left|\left\{n_{\alpha}\right\}\right\rangle$ and the operator identity can be formulated straightforwardly [see Eq. (B3)].
Therefore, the following operator identity is established:

$$
\begin{aligned}
& e^{-\beta \frac{1}{2} \sum_{\alpha, \beta} U_{\alpha \beta} \hat{n}_{\alpha} \hat{n}_{\beta}} \\
& \quad=\int d[\tilde{\boldsymbol{\varphi}}] e^{-\frac{\beta}{2} \sum_{\alpha \beta} \tilde{\varphi}_{\alpha}\left(U^{-1}\right)_{\alpha \beta} \tilde{\varphi}_{\beta}-i \beta \sum_{\alpha} \tilde{\varphi}_{\alpha} \hat{n}_{\alpha}} .
\end{aligned}
$$

The path-integral representation of the Hubbard-Stratonovich transformation should be modified accordingly to be consistent with this operator identity.

The situation gets tricky when one treats a term involving mutually noncommuting operators. A common example is the spin exchange term $\hat{\boldsymbol{S}}^{2}$, which one sometimes tries to decompose in a spin-rotational way using the HubbardStratonovich transformation. The decomposition relies on the integral identity

$$
e^{\beta J S^{2}}=\int d[\boldsymbol{m}] e^{-\beta \frac{m^{2}}{4 J}-\beta \boldsymbol{m} S},
$$

where $\boldsymbol{m}$ refers to a three-component vector that obeys the Gaussian distribution respectively and $d[\boldsymbol{m}]$ includes the normalization. We emphasize that though the above identity is correct for any vector $\boldsymbol{S}$, one cannot promote it to an operator identity with the spin operator $\hat{S}$, because of its noncommutative nature. One can easily check this fact by taking the trace of both sides of Eq. (D2) for spin one-half operator: The left-hand side yields $2 e^{\frac{3}{4}} \beta J$, whereas the right-hand side yields $2 e^{\frac{\beta J}{4}}(1+\beta J / 2)$. Therefore, applying such types of the HS decoupling involving noncommutative operators should be cautioned in the path-integral formulation.
[1] R. P. Feynman and A. R. Hibbs, Quantum Mechanics and Path Integrals (McGraw-Hill, New York, 1965).

[2] H. Kleinert, Path Integrals in Quantum Mechanics, Statistics, Polymer Physics, and Financial Markets, 5th ed. (World Scientific, Singapore, 2009).

[3] J. R. Klauder and B.-S. Skagerstam, Coherent States: Applications in Physics and Mathematical Physics (World Scientific, Singapore, 1985).

[4] W.-M. Zhang, D. H. Feng, and R. Gilmore, Rev. Mod. Phys. 62, 867 (1990).

[5] A. Altland and B. Simons, Condensed Matter Field Theory, 2nd ed. (Cambridge University Press, Cambridge, UK, 2010).

[6] J. H. Wilson and V. Galitski, Phys. Rev. Lett. 106, 110401 (2011).

[7] G. Kordas, S. I. Mistakidis, and A. I. Karanikas, Phys. Rev. A 90, 032104 (2014).

[8] P. Phillips, Rev. Mod. Phys. 82, 1719 (2010).

[9] P. A. Lee, N. Nagaosa, and X.-G. Wen, Rev. Mod. Phys. 78, 17 (2006).

[10] H. Kleinert, Electron. J. Theoret. Phys. 8, 57 (2011); H. Kleinert, Particles and Quantum Fields (World Scientific, Singapore, 2016), Sec. 14.15.
[11] L. P. Kadanoff and G. Baym, Quantum Statistical Mechanics (Addison-Wesley, New York, 1962).

[12] L. V. Keldysh, Zh. Eksp. Teor. Fiz. 47, 1515 (1965) [Sov. Phys. JETP 20, 1018 (1965)].

[13] J. Rammer, Quantum Field Theory of Non-equilibrium States (Cambridge University Press, Cambridge, UK, 2007).

[14] A. Kamenev, Field Theory of Non-equilibrium Systems (Cambridge University Press, Cambridge, UK, 2011).

[15] A. Kamenev and Y. Gefen, Phys. Rev. B 54, 5428 (1996).

[16] H. Kleinert, Ann. Phys. 253, 121 (1997).

[17] S. Florens and A. Georges, Phys. Rev. B 66, 165111 (2002); S. Florens, P. San José, F. Guinea, and A. Georges, ibid. 68, 245311 (2003).

[18] N. Sedlmayr, I. V. Yurkevich, and I. V. Lerner, Europhys. Lett. 76, 109 (2006).

[19] R. Lynch, Phys. Rep. 256, 367 (1995).

[20] I. L. Aleiner, P. W. Brouwer, and L. I. Glazman, Phys. Rep. 358, 309 (2002).

[21] G.-L. Ingold and Y. Nazarov, Charge tunneling rates in ultrasmall junctions, in Single Charge Tunneling, NATO ASI Series Vol. 294, edited by H. Grabert and M. Devoret (Springer, New York, 1992), Chap. 2, pp. 21-107.

[22] H. Schoeller and G. Schön, Phys. Rev. B 50, 18436 (1994). 